\title{
Computer Vision-based Early Fire Detection Using Enhanced Chromatic Segmentation and Optical Flow Analysis Technique
}

\author{
Arnisha Khondaker ${ }^{1}$, Arman Khandaker ${ }^{1}$, and Jia Uddin ${ }^{2}$ \\ ${ }^{1}$ Department of Computer Science and Engineering, BRAC University, Bangladesh \\ ${ }^{2}$ Department of Technology Studies, Woosong University, South Korea
}

\begin{abstract}
Recent advances in video processing technologies have led to a wave of research on computer vision-based fire detection systems. This paper presents a multi-level framework for fire detection that analyses patterns in chromatic information, shape transmutation, and optical flow estimation of fire. First, the decision function of fire pixels based on chromatic information uses majority voting among state-of-the-art fire color detection rules to extract the regions of interest. The extracted pixels are then verified for authenticity by examining the dynamics of shape. Finally, a measure of turbulence is assessed by an enhanced optical flow analysis algorithm to confirm the presence of fire. To evaluate the performance of the proposed model, we utilize videos from the Mivia and Zenodo datasets, which have a diverse set of scenarios including indoor, outdoor, and forest fires, along with videos containing no fire. The proposed model exhibits an average accuracy of $97.2 \%$ for our tested dataset. In addition, the experimental results demonstrate that the proposed model significantly reduces the rate of false alarms compared to the other existing models.
\end{abstract}

Keywords: Fire detection, color segmentation, shape analysis, optical flow analysis, Lucas-Kanade tracker, neural network.

Received September 26, 2019; accepted March 17, 2020

https://doi.org/10.34028/iajit/17/6/13

\section{Introduction}

Fire is a common disaster that claims thousands of lives and does insurmountable damage to property every year. Early fire detection has, therefore, become increasingly important to protect against this hazard. Even though sensor-based fire detection systems relying on heat or smoke signals are ubiquitous, these systems have multiple drawbacks. First, sensor-based detectors have to be densely distributed because they have limited coverage in terms of area. This results in raised expenses for both installation and maintenance [4]. Second, heat and smoke do not spread instantaneously [12], making the sensors intrinsically slow at picking up these signals from the environment. To overcome these challenges, computer vision-based fire detection techniques have been gaining traction over the last couple of years $[4,7]$.

Cutting-edge computer vision algorithms utilizing layers of pattern recognition can make feasible early detection of fire $[6,9,11]$. For accurate detection, these layers attempt to analyse various distinctive features of fire, which include its characteristic color, shape, flickering rate, and growth, and the spatiotemporal difference in frame sequences. This paper presents a method of (Luminance (Y), Blue-luminance (U), Redluminance (V)) (YUV) color segmentation, a generic shape analysis technique, and enhanced optical flow analysis of candidate fire regions for robust detection of fire.
The remainder of this paper is organized as follows. Related works are overviewed in section 2 , and the proposed model is illustrated in detail in section 3 . Experimental results are presented in section 4, and the paper is concluded in section 5 .

\section{Related Works}

In the last few decades, several methods have been proposed to detect fire in videos acquired by traditional video surveillance cameras. Some form of color evaluation is common to all models of fire detection. Color information can be extracted using chromatic segmentation algorithms that rely on multiple color spaces, such as Red Green Blue (RGB) [7, 13], YCbCr (Green (Y), Blue (Cb), Red (Cr)) [3, 6], L*a*b* [4], YUV [9], Hue-Saturation-Value (HSV), or even a blend of different color spaces [16].

Chen et al. [5] employed a statistical color model and used the intensity and saturation information of the red component in conjunction with a binary background mask with median filtering. The method uses statistical values of chromatic information and is straightforward to understand and implement. Variations of the formulae originally proposed by Chen are presented in a vast body of literature. Celik derived and developed new rules based on $\mathrm{YCbCr}$ [4]. These were later altered and utilized in [3]. Chen and Huang [8] modeled their works on HSV, and 
Seebamrungsat et al. [16] implemented their techniques in both $\mathrm{HSV}$ and $\mathrm{YCbCr}$ color spaces. A probabilistic approach based on YUV color space is exploited in [1], where the thresholding of potential fire pixels relies on a support vector machine instead of a naïve heuristic.

However, detectors based on color analysis fail to consider other important characteristics of fire, such as shape, growth and flickering. It is easy to trigger the chromatic filter by secondary luminous sources like light bulbs in the scene or variation in the luminescence of the scene. To reduce the number of false-positive, other features of fire must be taken into account. Previously, frame differencing [16] and image segmentation based on colour analysis $[6,9,13]$ were used. In [10], Gaussian mixture models are used to extract the moving foreground from the static background. Although a Gaussian mixture model has high accuracy, it is too expensive computationally, which makes it unsuitable for real-time fire detection.

Khan et al. [11] used a novel foreground extraction technique based on the rapidly changing value of the blue channel in fire pixels. Foggia et al. [9] used an unusual multi-expert system with a novel motion descriptor that adopts a bag-of-words approach commonly observed in Natural Language Processing (NLP) algorithms. Another technique popularly used to detect the temporal behaviour of flame boundaries is wavelet transformation [4, 17]. However, to gain satisfactory results from wavelet analysis, the frame rate must be sufficiently high, limiting its applicability. Other feasible approaches include optical flow analysis as carried out in [15] and enhanced in [14]. This has been observed to be highly effective in separating the uniform motion of rigid objects from the disorderly movement of fire plumes. However, a static threshold of 2 for optical flow complexity was used in [15], and the overall performance was not satisfactory with a more diverse dataset.

\section{Proposed Model}

The proposed model utilizes three major phases for the detection of fire. An overview of the model is illustrated in Figure 1.

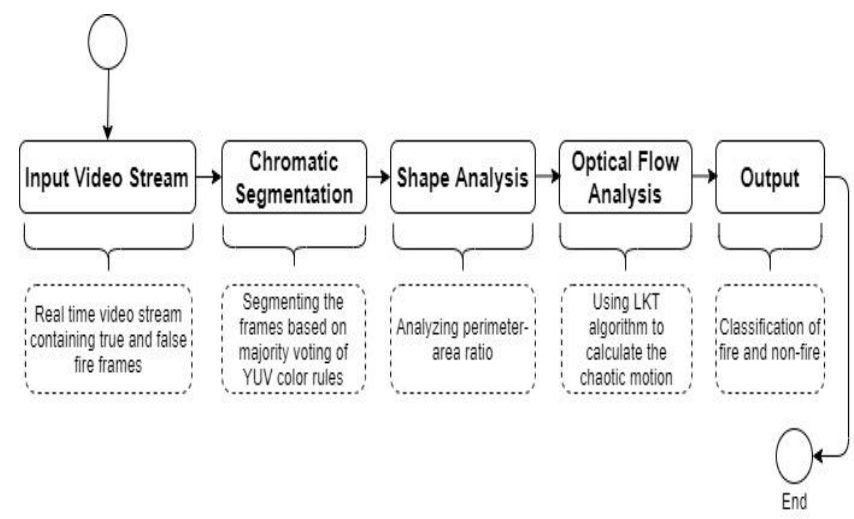

Figure 1. Workflow of the proposed model displaying the different phases and their brief descriptions.

\subsection{Chromatic Segmentation in YUV Color Space}

The non-chemical fire has a characteristic color that almost always appears in the red-yellow range. Chromatic segmentation based on this distinctive feature thus naturally acts as the primary trigger for fire detection. Among the existing segmentation methods, many are well-formulated using principled techniques of image processing, while others are based on heuristic thresholds and empirical observation. Based on the experimental analysis, pixels in most flames exhibit a Red channel value greater than that of Green, as well as a Green channel value greater than that of Blue [3]:

$$
R(i, j) \geq G(i, j)>B(i, j)
$$

Where $R(i, j), G(i, j)$, and $B(i, j)$ are the Red, Green, and Blue (RGB) channel values of pixel $(i, j)$ respectively.

We have used YUV color space as it considers the chrominance and luminance of a pixel [4]. The constraints in [4] can be equivalently expressed in the YUV plane by converting from RGB to YUV using the well-known conversion rules specified in [17]. Based on the converted output, the following rules can be used to define a fire pixel, so that we obtain the following generic pixel $(x, y)$ of the image:

$$
\begin{aligned}
& r_{1}(x, y)=\{1 \text { if } Y(x, y)>U(x, y) \\
& r_{2}(x, y)= \begin{cases}1 & \text { if } V(x, y)>U(x, y) \\
0 & \text { otherwise }\end{cases}
\end{aligned}
$$

Where $r_{n}(x, y)$ indicates any pixel in spatial location $(x, y)$, which takes the value of 1 if it exhibits fire-like color and 0 otherwise. $Y(x, y, U(x, y)$, and $V(x, y)$ are the $Y, U$, and $V$ channel values in spatial location $(x, y)$, respectively.

Moreover, it is empirically demonstrated that the $Y$ component of fire pixels is generally larger than the mean $Y$ component in a frame. Similar rules [9] can be equivalently expressed as follows:

$$
\begin{gathered}
r_{3}(x, y)=\{1 \text { if } Y(x, y)>\text { Ymean } \\
0, \text { otherwise }\} \\
r_{4}(x, y)=\{1 \text { if } U(x, y)<\text { Umean } \\
0, \text { otherwise }\} \\
r_{5}(x, y)=\{1 \text { if } V(x, y)>\text { Vmean } \\
0, \text { otherwise }\}
\end{gathered}
$$

Likewise, the mean values of the three channels in YUV color space for an $M x N$ image can be defined as follows:

$$
\begin{aligned}
\mathrm{Y}_{\text {mean }} & =\left(\frac{1}{\mathrm{M} * \mathrm{~N}}\right) \cdot \sum_{\mathrm{i}=0}^{\mathrm{M}} \sum_{\mathrm{j}=0}^{\mathrm{N}} \mathrm{Y}(\mathrm{i}, \mathrm{j}) \\
\mathrm{U}_{\text {mean }} & =\left(\frac{1}{\mathrm{M} * \mathrm{~N}}\right) \cdot \sum_{\mathrm{i}=0}^{\mathrm{M}} \sum_{j=0}^{\mathrm{N}} \mathrm{U}(\mathrm{i}, \mathrm{j}) \\
\mathrm{V}_{\text {mean }} & =\left(\frac{1}{\mathrm{M} * \mathrm{~N}}\right) \cdot \sum_{\mathrm{i}=0}^{\mathrm{M}} \sum_{j=0}^{\mathrm{N}} \mathrm{V}(\mathrm{i}, \mathrm{j})
\end{aligned}
$$


Where $Y(i, j), U(i, j)$, and $V(i, j)$ are the $Y, U$, and $V$ components of a pixel in spatial locatisson $(i, j)$ in YUV color space, respectively.

Furthermore, it has been shown that fire pixels show a significant difference between $U$ and $V$ components [3].

$$
|V(x, y)-U(x, y)| \geq t_{c}
$$

Where $t_{c}$ is the threshold that must be exceeded.

However, in the experimental analysis, it was observed that Equation (10) works poorly in the case of relatively brighter flames. Therefore, in this paper, Equation (10) was modified into Equations (11) and (12) to eliminate the threshold value.

$$
\begin{gathered}
r_{6}(x, y)=\{1 \text { if }(0.025 * V(x, y)-0.025 * U(x, y)-1)>0 \\
0, \text { otherwise }\} \\
r_{7}(x, y)=\left\{1 \text { if } \left(\begin{array}{c}
0.025 * U(x, y)-0.025 * V(x, y)-1)>0 \\
0, \text { otherwise }\}
\end{array}\right.\right.
\end{gathered}
$$

Figure 2 illustrates the improved performance of the split rules in detecting a fire region compared to the existing Equation (10).

A common limitation of chromatic filtering algorithms [2] is that they perform logical conjunction of all the rules to segment out the region of interest. This approach often fails to detect visually distant flames. To overcome this limitation, a more liberal approach is undertaken in the proposed system by taking a vote on all the rules and segmenting the image based on the majority decision, as described in Equation (13).

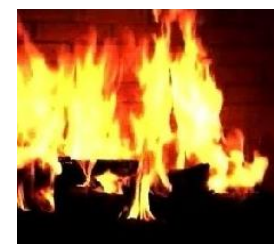

a) The original frame of a bright flame at close proximity.

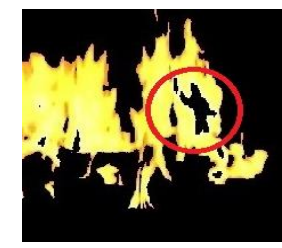

b) Segmentation using Equation (10)

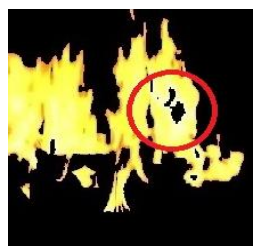

c) Segmentation using Equations (11) and (12).
Figure 2. Comparison showing improved accuracy of the enhanced equations in Red squares.

$$
\begin{gathered}
\varphi_{M}(x, y)=\sum_{k=1}^{7} \mathrm{r}_{k}(x, y) \\
F_{\text {candidate }}(x, y)=\left\{1 \text { if } \varphi_{M}(x, y) \geq M\right. \\
0, \text { otherwise }\}
\end{gathered}
$$

Where $M$ denotes the number of rules that must give a positive indication. $\varphi_{M}(x, y)$ is a measure of the number of rules applied to pixel $(x, y)$ as a fire pixel. $F_{\text {candidate }}(x, y)$ is the candidate fire pixel $(x, y)$, which is 1 if it is indeed a fire pixel and 0 otherwise

The smaller is the value for $M$ in Equation (13), the larger is the number of false positives. The value is therefore chosen such that any potential fire pixel is not ignored by being too restrictive. By empirical study, in this paper, the value of $M$ was adjusted to be 4 . Additionally, the shape and optical flow analyses are designed to address this issue. Figure 3 shows that the

majority decision rule can extract fire regions more accurately.

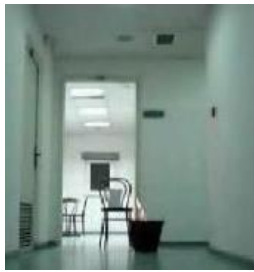

a) A frame from a video with a fire generated in a bucket in indoor conditions.

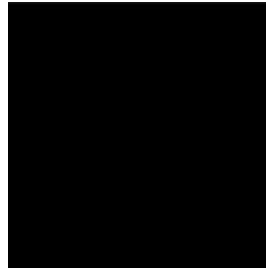

b) The binary mask of (a) without application of the majority voting rule specified in Equation (13).

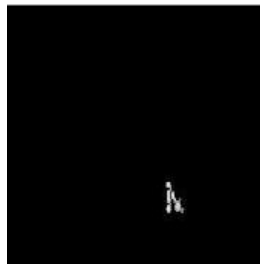

c) Shows the binary mask of (a) when the majority voting rule is applied.
Figure 3. Comparison between the binary masks of a sample frame.

\subsection{Shape Analysis}

A distinguishing feature of flames is their complex shape that varies rapidly as a function of time. As suggested in [9], blobs from two consecutive frames from the previous step (section 3.1) are identified, and the ratio of their perimeter and area is calculated, which can be described as follows:

$$
R_{t}=\frac{P_{t}}{A_{t}}
$$

Where $R_{t}$ is the ratio of perimeter $P_{t}$ and area $A_{t}$.

This ratio for the fire blobs is considerably larger than the ratio calculated for ordinary objects that manage to slip through the previous step. For further substantiation, the ratio for a blob detected in frame $t$ is compared with the nearest blob detected in the next frame $t+1$. This can be stated as follows:

$$
S_{t v}=\frac{r_{t}-r_{t+1}}{r_{t}}
$$

Where $S_{t v}$ denotes the change in shape.

If $S_{t v}$ in Equation (15) is higher than the given threshold $t$, this step classifies the frame as containing the fire. In this paper, by empirical study, $t$ was adjusted to be 0.4 for our dataset. This phase of the model is intended to remove ordinary non-fire objects exhibiting the color of fire.

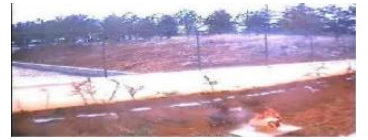

a) Sample frame at time $t$

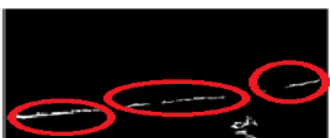

c) Segmented frame before performing shape analysiswith the spurious nonmoving regions marked in red circles.

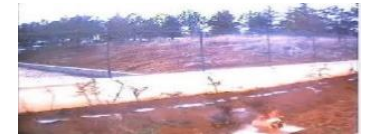

b) Frame at time $t+1$.

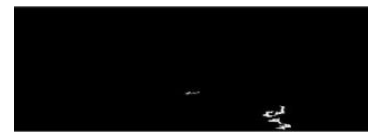

d) Segmented frame after shape analysis with thespurious regions eliminated.
Figure 4. Output of two consecutive frames from a video containing both fire and non-fire regions exhibiting the characteristic color of fire. 
Figure 4 presents the result of the shape analysis of a sample scenario. Most of the non-growing regions were eliminated due to the shape and growth analyses.

\subsection{Optical Flow Analysis}

Fire is a turbulent physical phenomenon, and the inner edges of fire plumes exhibit a chaotic displacement as a function of time. This feature is so unique to fire that successful extraction results in a dramatic reduction of false positives. This feature can be extracted using optical flow tracking algorithms. In the proposed system, an enhanced Lucas-Kanade optical flow analysis technique is suggested to calculate the turbulence of fire [15].

The Lucas-Kanade Tracking (LKT) algorithm uses a set of corners to track a sequence of frames. At first, the Features from the Accelerated Segment Test (FAST) algorithm are utilized to detect 50 corners in the firelike regions. These corners are subsequently fed into the Lucas-Kanade tracker to be tracked across a sequence of frames. The LKT algorithm then calculates and returns a set of motion velocity vectors for each of these corners from frame $t$ to frame $t+1$. The vectors can be represented as follows:

$$
\begin{aligned}
& p=\left[p_{x}, p_{y}\right]_{i}, i=0,1,2, \ldots, n \\
& q=\left[q_{x}, q_{y}\right]_{i}, i=0,1,2, \ldots, n
\end{aligned}
$$

Where $p$ and $q$ denote the starting and ending points, respectively, of a corner from frame $t$ to frame $t s+1$, and $n$ indicates the number of corners.

The result of the LKT algorithm on a set of corners is depicted in Figure 5. The velocity vectors of the corners detected in fire are represented using purple lines. The longer lines indicate faster movement of the corner.

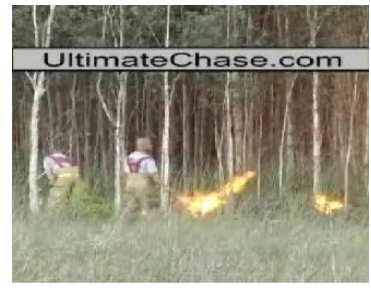

a) Original frame from a video with fire in outdoor conditions. b) The velocity vectors calculated from the corners detected in the flame region in (a).

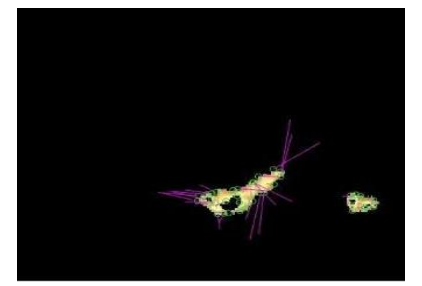

Figure 5. Velocity vectors obtained from a frame containing forest fire.

We can calculate the average flow rate of fire using the vectors calculated in Equations (16) and (17), which gives a rough measure of complexity. The average flow rate is calculated using the following Equation:

$$
F=\frac{1}{n} \sum_{k=0}^{n} \sqrt{\left.\left(p_{y k}-q_{y k}\right)^{2}+\left(p_{x k}-p_{x k}\right)^{2}\right)}
$$

Where $n$ denotes the number of corners being tracked, and $F$ is the average flow rate.

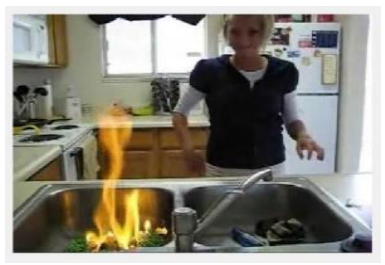

a) The original frame of fire plumes at close vantage point (scene 1).

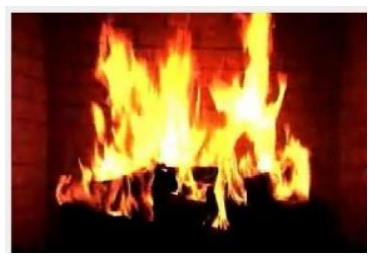

c) The original frame of fire plumes at close vantage point (scene 2).

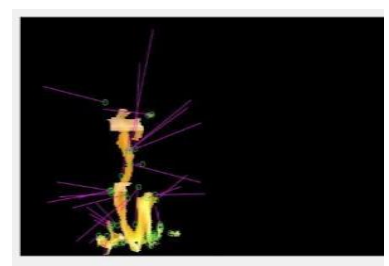

b) Corresponding velocity vectors for (a).

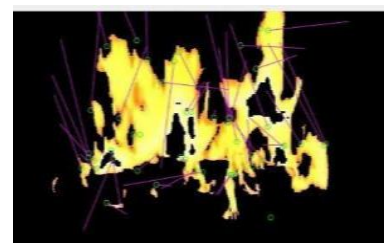

d) Corresponding velocity vectors for (c).
Figure 6. Velocity vectors of a frame containing indoor fire plumes.

This flow rate complexity represents the chaotic, non-deterministic motion of the local features of fire. Consequently, the value of this will differ between fire and non-fire objects. The proposed model makes use of this average flow rate complexity to analyze the possibility of fire. In the empirical study, the value of $n$ in Equation (18) has been concretized to be 10, and the complexity is calculated $n$ times. Every time the complexity exceeds the threshold 0.5 , a counter is incremented. If the complexity exceeds the threshold more times than not in $n$ attempts, the scene is considered a potential fire scene. The average flow rate values for 1000 such $n$-frame scenes are then extracted and subsequently trained using a scaled conjugate gradient back-propagation neural network.

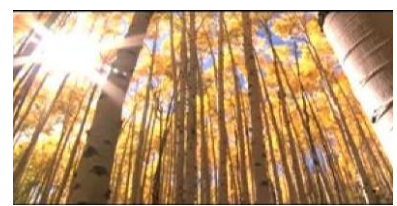

a) The original frame from a video with sunlight shimmering through a forest containing trees and leaves having the characteristic color of fire.

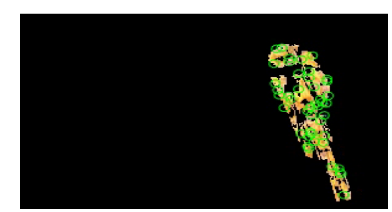

b) The corresponding motion vectors obtained from (a).
Figure 7. Motion vectors of a non-fire scene with fire-like properties.

To test the efficacy of the model, the $n$-frames scene is fed to the neural network for analysis, which then attempts to classify the $n$-frames scene as a fire or non-fire scene. The model successfully demonstrates promising performance in picking up a pattern from the flow rate complexity. Figure 6 illustrates the velocity vectors of a sample fire, where the vectors of fire are quite dispersing and chaotic.

The turbulent motion vectors in the fire scenes depicted in Figure 6 are easily distinguishable from the relatively calm motion vector in the non-fire scene in Figure 7. 


\section{Experimental Results}

To evaluate the performance of the proposed model as a simulation tool, we utilized MATLAB and tested it using a diverse dataset containing videos in a wide variety of scenarios. The dataset includes videos containing the fire at different illumination levels and videos with ordinary objects that display fire-like color. The dataset was prepared predominantly using videos from the Mivia [9] dataset with some videos from Zenodo, and the rest were taken from YouTube or recorded personally. The videos can be broadly divided into three categories: indoor, outdoor, and forest fires.

The proposed model yields a comparatively greater true positive rate in scenarios where the fire is situated in a distant region. It excels over the competing models in these cases because these models employ a stricter set of color segmentation rules in their first phase of analysis. The restrictive color rules fail to extract the small fire regions in the scenarios where the fire is located far distant. Consequently, the comparatively liberal color rules employed in the proposed model yield a better true positive rate.

The results of the enhanced segmentation rules that yield this better accuracy of True Positive (TP) in percentage (\%) are presented in Tables 1 and 2.

Table 1. Comparison of the performance of enhanced Equations (11) and (12) versus traditional Equation (10).

\begin{tabular}{|c|c|c|}
\hline Case & Average TP & Average FP \\
\hline Using Equation (10) & 93.0 & 2.6 \\
\hline Using enhanced Equations (11) and (12) & 97.7 & 2.9 \\
\hline
\end{tabular}

Table 2. Comparison of the performance of the majority voting rule in Equation (13).

\begin{tabular}{|c|c|c|}
\hline Case & Average TP & Average FP \\
\hline Without using Equation (13) & 82.2 & 8.7 \\
\hline Using Equation (13) & 98.9 & 18.0 \\
\hline
\end{tabular}

The average TP rate is the percentage of fire pixels correctly identified in a scene, whereas the average False Positive (FP) rate is the percentage of non-fire pixels misidentified as fire. Enhanced Equations (11) and (12) identify a greater number of true fire pixels compared to Equation (10). This test was carried out against frames containing very bright flames. Even better performance is provided by the majority voting rule in Equation (13), which identifies $98.9 \%$ of fire pixels correctly. This test was carried out against frames containing flames situated far away. Although it simultaneously increases the average FP rate, the effect of this is eliminated by the optical flow analysis phase.

Additionally, the performance of the proposed model with enhanced color rules and an optical flow analysis technique is compared with the model presented by Khan et al. [11] and Rinsurongkawong et al. [15]. Assessment is performed concerning the rates of true positives and false positivesin a scene of 10 frames, and the corresponding model's capability to correctly classify the 10 -frame scene as a fire scene or non-fire scene.

Table 3 shows the comparison of the models in different environments, which reveals that the proposed model yields an overall average accuracy of 97.2\%, outperforming both Khan's et al. [11] and Rinsurongkawong's et al. [15] models. Furthermore, the proposed model achieved greater success in the reduction of false positives, demonstrating an only $2.21 \%$ false-positive rate compared to $7.78 \%$ and $6.76 \%$ for Khan's et al. [11] and Rinsurongkawong's et al. [15] models, respectively.

Table 3. Comparison of the models exposed to a diverse set of scenarios.

\begin{tabular}{|c|c|c|c|c|c|c|}
\hline & $\begin{array}{c}\text { Khan } \text { et al. } \text { [11] } \\
\text { (in \%) }\end{array}$ & $\begin{array}{c}\text { Rinsurongkawong } \text { et al. } \\
{[15]}\end{array}$ & \multicolumn{2}{|c|}{$\begin{array}{c}\text { Proposed Model } \\
\text { (in \%) }\end{array}$} \\
\hline Class & TP & FP & TP & FP & TP & FP \\
\hline Indoor & 96.04 & 5.84 & 96.98 & 14.46 & 99.06 & 2.1 \\
\hline Outdoor & 90.4 & 3.94 & 91.76 & 2.84 & 94.6 & 3.14 \\
\hline Forest & 99.73 & 17.43 & 99.43 & 0.47 & 98.33 & 0.83 \\
\hline Overall & 94.7 & 7.78 & 95.5 & 6.76 & 97.2 & 2.21 \\
\hline
\end{tabular}

Khan's et al. [11] model is typically weaker when there is sunlight in the background. Khan et al. [11] uses a novel foreground extraction technique that exploits the flickering property of fire by detecting the rapidly changing blue channel value of fire pixels. However, this property is also true for the pixels in sunlight, yielding an alarming rate of false positives in scenes containing sunlight. The proposed model, in contrast, does not suffer from this weakness due to its reliance on dynamic optical flow analysis, which yields different results for fire and sunlight.

Similar to the proposed model, Suchet's [15] model also does not suffer from the limitation of Khan's model. However, Suchet's [15] model is weaker when objects exhibiting the characteristic color of fire are moved in a haphazard, unpredictable way. Suchet [15] employs an optical flow analysis technique that calculates the variation of optical flow over time. However, the model uses a naïve thresholding approach that fails to differentiate the unpredictable motion of fire from the unpredictable motion of an object moving in a non-deterministic way.

Alternatively, the proposed model calculates the average flow rate of candidate pixels and employs a back-propagating neural network to identify a pattern from the average flow rate, which reduces the false positives rate. Overall, the model is powerful in its ability to detect fire in all types of scenarios. It performs equally well in detecting indoor, outdoor, and forest fires at different levels of illumination and varying levels of distance from the camera. Moreover, compared to the competing models, it increases the true positive rate of detection and significantly reduces the false positive rate. 


\section{Conclusions and Future Work}

A robust fire detection system has been proposed in this paper which analyzes several properties of fire, including color, shape, and turbulence. An enhanced chromatic segmentation algorithm using a majority voting system to detect $F_{\text {candidate }}(x, y)$ if $\varphi_{M}(x, y)>=$ 4has been adopted in cohesion witha generic shape analysis technique to detect two of the most fundamental visual properties of fire. As the final and most important step of the model, an enhanced LKT optical flow analysis algorithm was proposed to detect the disorderly motion of fire plumes. Otherwise, the rate of false-positive would be higher. The proposed model was tested using a diverse dataset, and it outperformed existing models by exhibiting an average accuracy of $97.2 \%$.

Future work will be intended to carry out the optical flow analysis step using a hybrid neural network that is trained directly with a set of principled turbulence values as the feature vector.

\section{References}

[1] Benezeth Y., Jodoin P., Emile B., Laurent H., and Rosenberger C., "Comparative Study of Background Subtraction Algorithms," Journal of Electronic Imaging, vol. 19, no. 3, 2010.

[2] Celen V. and Demirci M., "Fire Detection in Different Color Models," in Proceedings WorldComp, Las Vegas, 2012.

[3] Çelik T. and Demirel H., "Fire Detection in Video Sequences Using A Generic Color Model," Fire Safety Journal, vol. 44, no. 2, pp. 147-158, 2009.

[4] Çelik T., "Fast and Efficient Method for Fire Detection Using Image Processing," ETRI Journal, vol. 32, no. 6, pp. 881-890, 2010.

[5] Chen J., He Y., and Wang J., "Multi-Feature Fusion-Based Fast Video Flame Detection," Building and Environment, vol. 45, no. 5, pp. 1113-1122, 2010.

[6] Corraya S. and Uddin J., "An Efficient Method for Detecting Electrical Spark and Fire Flame from Real-Time Video," in Proceedings of $3^{\text {rd }}$ International Symposium on Signal Processing and Intelligent Recognition Systems, Manipal, pp. 359-368, 2018.

[7] Chen T., Wu P., and Chiou Y., "An Early FireDetection Method Based on Image Processing," in Proceedings International Conference on Image Processing, Singapore, pp. 1707-1710, 2004.

[8] Chen L. and Huang W., "Fire Detection Using Spatial-Temporal Analysis," in Proceedings of the World Congress on Engineering, London, pp. 3-5, 2013.

[9] Foggia P., Saggese A., and Vento M., "Real-Time Fire Detection for Video-Surveillance
Applications Using a Combination of Experts Based on Color, Shape, and Motion," IEEE Transactions on Circuits and Systems for Video Technology, vol. 25, no. 9, pp. 1545-1556, 2015.

[10] Han X., Jin J., Wang M., Jiang W., Gao L., and Xiao L., "Video Fire Detection Based on Gaussian Mixture Model and Multi-Color Features," Signal, Image and Video Processing, vol. 11, no. 8, pp. 1419-1425, 2017.

[11] Khan R., Uddin J., and Corraya S., "Real-Time Fire Detection Using Enhanced Color Segmentation and Novel Foreground Extraction," in Proceedings $4^{\text {th }}$ International Conference on Advances in Electrical Engineering, Dhaka, pp. 488-493, 2017.

[12] Kumar T. and Reddy K., "Technique for Burning Area Identification Using IHS Transformation and Image Segmentation," The International Arab Journal of Information Technology, vol. 12, no. 6A, pp. 764-771, 2015.

[13] Khan R., Uddin J., Corraya S., and Kim J., "Machine Vision-based Indoor Fire Detection Using Static and Dynamic Features," International Journal of Control and Automation, vol. 11, no. 6, pp. 87-98, 2018.

[14] Mueller M., Karasev P., Kolesov I., and Tannenbaum A., "Optical Flow Estimation for Flame Detection in Videos," IEEE Transactions on Image Processing, vol. 22, no. 7, pp. 27862797, 2013.

[15] Rinsurongkawong S., Ekpanyapong M., and Dailey M., "Fire Detection for Early Fire Alarm Based on Optical Flow Video Processing," in Proceedings $9^{\text {th }}$ International Conference on Electrical Engineering/Electronics, Computer, Telecommunications and Information Technology, Phetchaburi, 2012.

[16] Seebamrungsat J., Praising S., and Riyamongkol P., "Fire Detection in The Buildings Using Image Processing," in Proceedings $3^{\text {rd }}$ ICT International Student Project Conference, Nakhon Pathom, pp. 95-98, 2014.

[17] Shon D., Kang M., Seo J., and MyonKim J., Frontier and Innovation in Future Computing and Communications, Springer, 2014. 


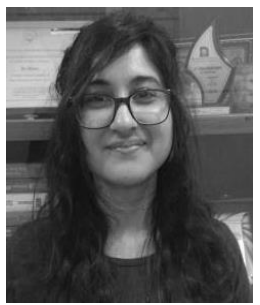

Arnisha Khondaker completed B.Sc. Degree in Computer Science and Engineering (CSE) from Brac University (BracU), Bangladesh in 2018. Currently, she is a Lecturer in the CSE department at BracU. Her research interests are computer vision and artificial intelligence.

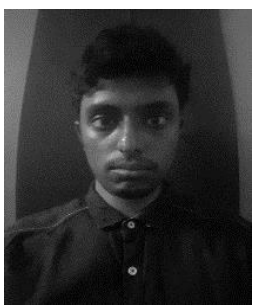

Arman Khandaker completed his B.Sc. Degree in CSE from BracU, Bangladeshin 2018. Currently he is working as a software engineer. His research interests include fire detection and computer vision.

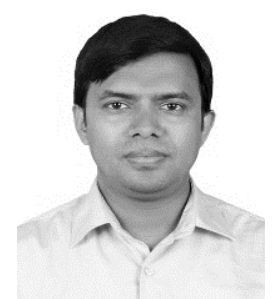

Jia Uddin received a BSc degree in Computer and Communication Engineering from International Islamic University Chittagong, Bangladesh in 2005, and an MSc. degree in Telecommunications from the Blekinge Institute of Technology, Sweden, in 2010. Hedid Ph.D. in Computer Engineering from the University of Ulsan, Korea, in January 2015. $\mathrm{He}$ is an Assistant Professor in Department of Technology Studies, Endicott College, Woosong University, South Korea and an Associate Professor (On Leave), Computer Science and Engineering Department at BracU, Bangladesh. His research interests include fault diagnosis, computer vision, and multimedia signal processing. 